Editorial

\title{
IS4SI SUMMIT Berkeley 2019 †
}

\author{
Marcin J. Schroeder ${ }^{1}$, GordanaDodig-Crnkovic ${ }^{2}$ and Mark Burgin ${ }^{3, *}$ \\ 1 Global Learning Center, Tohoku University, Sendai, Miyagi Prefecture, 980-8576, Japan; \\ schroeder.marcin.e4@tohoku.ac.jp \\ 2 Department of Computer Science and Engineering, Chalmers University of Technology, Gothenburg 40482, \\ Sweden; gordana.dodig-crnkovic@chalmers.se \\ 3 Department of Computer Science, University of California, Los Angeles (UCLA), Los Angeles, CA 90095, \\ USA; mburgin@math.ucla.edu \\ * Correspondence: mburgin@math.ucla.edu \\ † 2019 Summit of the International Society for the Study of Information, Berkeley, CA, USA, 2-6 June 2019.
}

Published: 12 May 2020

The leading theme of the 2019 Summit of the International Society for the Study of Information, held 2-6 June 2019 at the University of California at Berkeley, was the question "Where is the I in AI, and the meaning of Information?" This question addresses one of the central issues not only for scientific research and philosophical reflection, but also for technological, economic and social practice.

There is no doubt that the progress in designing computer based technological devices known as Artificial Intelligence (AI) systems has transformed the life and work of almost the entire human population. Moreover, this transformation continues and accelerates. At the same time, there is no one commonly accepted definition of AI. This is a reflection of the fact that there is no commonly accepted definition of intelligence in general. Moreover, there is no agreement about human intelligence, and psychologists and cognitive scientists frequently opt for multiple intelligences because, in essence, intelligence is a contextual trait. Thus, an important goal of researchers is to more definitely qualify the natural intelligence of people and maybe other living beings, making it possible to understand whether artificial intelligence, i.e., the intelligence of a machine, is the same, and how it is possible to estimate this intelligence.

In addition, there are also ethical issues in this area. AI is based on technology, and we can already see the great benefits of accelerating technological transformation, but also some of its negative, destructive or even dangerous consequences. In order to make the best use of already existing technologies and to avoid their negative consequences, as well as to assure the development of AI for good, we have to understand well their mechanisms and their interactions with humanity and natural environment. This is a surprisingly difficult task, especially for the situation when machines will be able to achieve superintelligence, i.e., higher intelligence than the intelligence of people.

The second part of the question, which was the leading theme of the Summit in Berkeley, was about the meaning of information. This reflects the actuality that intelligence is based on information and information processes, while meaning is a pivotal characteristic of information. The current situation is characterized by the common objection to the ascription of intelligence to currently existing technological devices due to the doubt that these artefacts are capable of associating meaning with formally encoded information. Of course, this brings us back to the centuries of philosophical discourse on the mind-body relationship, which is still an open question and an objective of research and philosophical reflection which, in the case of AI, is transformed into the problem of the mind-machine relationship. The contemporary achievements of science, mathematics and philosophy position these problems in the context of the Existential Triad of the world, which consists of three worlds: physical, mental and structural worlds. 
New achievements in the research in this area, reflecting different approaches to the described problems, were presented in the talks of the participants of the 2019 Summit, which contained many interesting ideas and appealing results.

The 2019 Summit was organized as an assembly of parallel conferences and workshops, which included on-site and off-site presentations. In this issue, we publish selected proceedings of the talks delivered at the following conferences:

- Conference Morphological, Natural, Analog and Other Unconventional Forms of Computing for Cognition and Intelligence (MORCOM) organized by Gordana Dodig-Crnkovic and Marcin J. Schroeder

- The Fourth International Conference on Philosophy of Information (POI) organized by Joseph Brenner and Kun $\mathrm{Wu}$

- Conference Theoretical Information Studies (TIS) organized by Mark Burgin

- Workshop on Habits and Rituals (HAR) organized by Raffaela Giovagnoli and Gianfranco Basti

Conflicts of Interest: The authors declare no conflict of interest.

(C) 2020 by the authors. Licensee MDPI, Basel, Switzerland. This article is an open access article distributed under the terms and conditions of the Creative Commons Attribution (CC BY) license (http://creativecommons.org/licenses/by/4.0/). 